\title{
Black in White - Magnetic in Paper
}

\author{
Shuai Xu \\ Producer \\ Goldsmiths, London, SE14 6NW \\ Sxu003@gold.ac.uk
}

\section{INTRODUCTION}

Black in White - Magnetic in Paper is an interactive installation engaging with people's hand gestures, which using materials paired with technology. The piece was trying to convert traditional Chinese landscape ink painting to computational form, by focusing on simple intangible interactions to representation complex tangible "Xie Yi" (Freehand) painting.

The charm and life of Chinese landscape paintings is not only the external appearance of the paper but also the invisible, sensible rhythm beyond the pen and ink. However, the traditional form of Chinese landscape painting enables the artist to express their emotions through brushstroke on paper. Most of artist's emotions are shown in a freehand way, and the penetration or spread of ink on rice paper sometimes reveals a surprising style. My work changed the traditional material ink by replacing it with magnetic powder. During the movement of the magnetic powder, traces are left on the rice paper; this is a new portrayal expression of the material. At the same time, the leap motion controls the movement of the magnetic powder according to the gesture of observers. I hope that my work can redefine people's feelings about Chinese landscape painting and observe its charm in black and white.

\section{CONCEPT AND BACKGROUND RESEARCH}

\subsection{Chinese landscape painting}

Many critics consider landscape to be the highest form of Chinese painting. Early landscape first appeared as part of figure painting, providing the natural setting for story narration. Images of nature have remained a potent source of inspiration for artists up to the present day. While Chinese landscape painting has been transformed by a millenary of human title, Chinese artistic expression has also been deeply influenced with forms of the natural world. When viewing Chinese landscape paintings, Chinese depictions of nature appear to be mere representations of the external world; however, they are expressions of the minds and hearts of the individual artists - cultivated landscapes that embody the culture and cultivation of their masters.

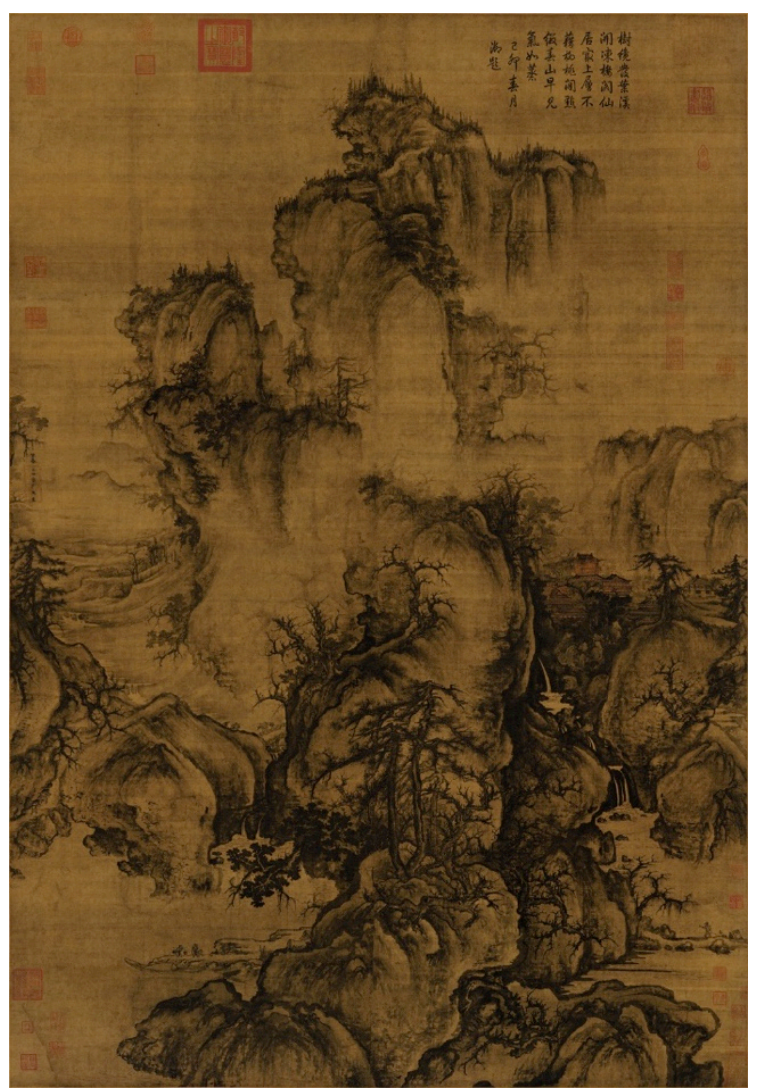

Figure 1: Early Spring (早春圖)Guo Xi (郭熙, active 11th century), Song Dynasty (960-1279).

\subsection{Narrative materials}

A New method, called narrative materials, is new ways of articulation through imagery and material creation for new sensory perceptions. Designing material-based languages aim to provide an aesthetic impetus on research methods and questions to arise with unrestrained curiosity and undisciplined search within multidisciplinary gatherings. 


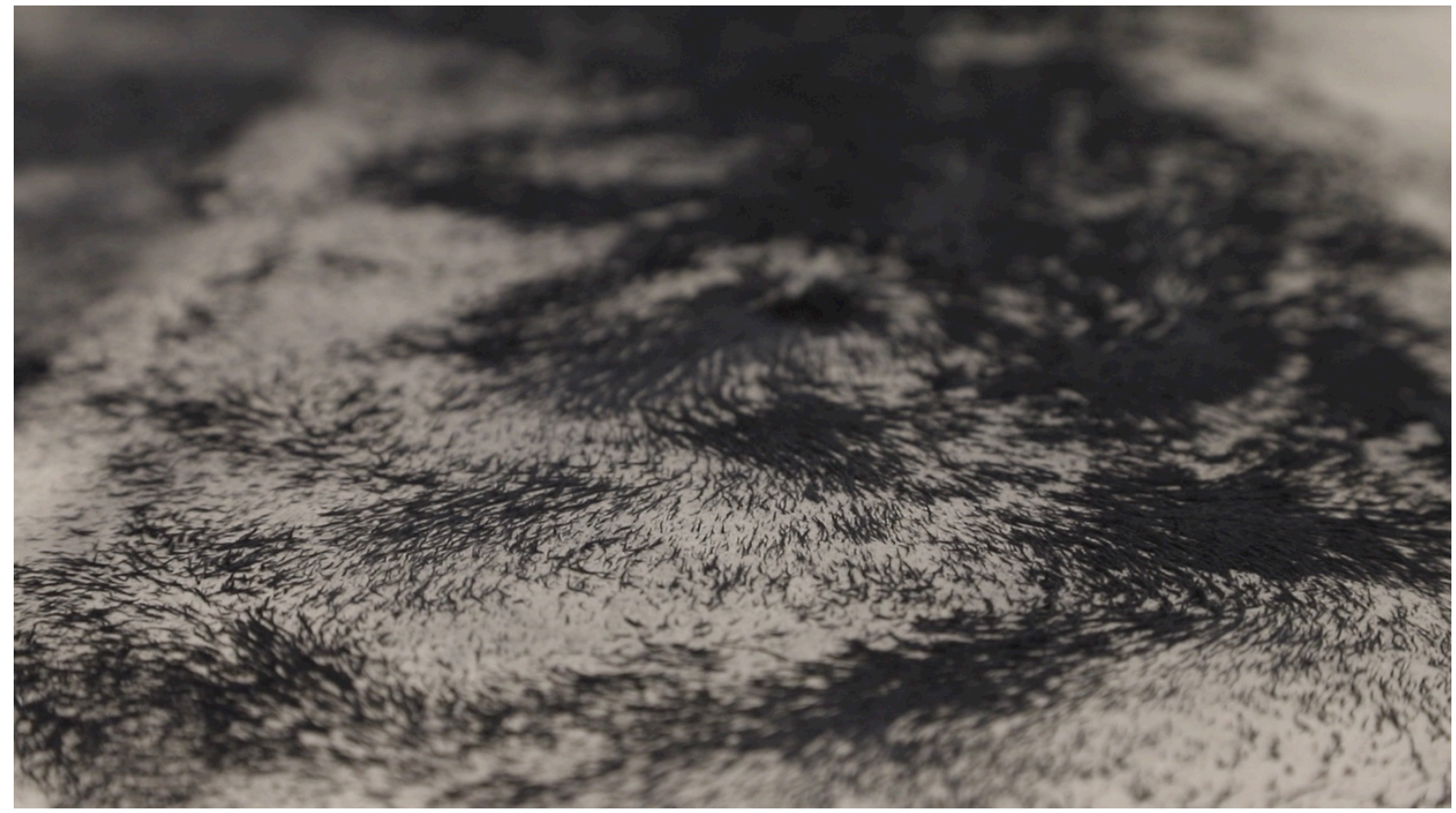

Figure 2: User painting

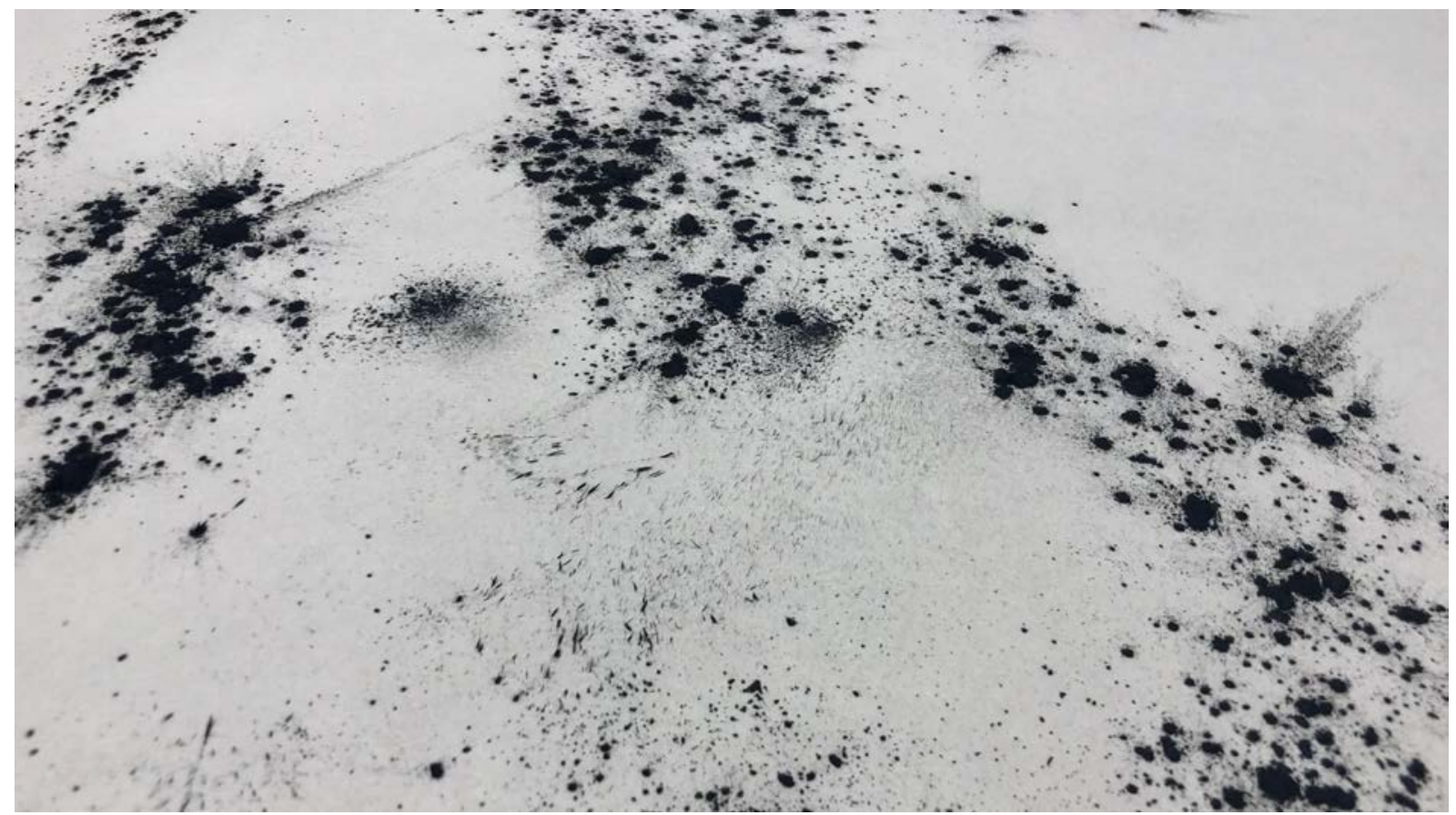

Figure 3: User painting

\subsection{Material representation}

Choosing the right materials to complete my project has been a challenge since we began our research. In the field of computational arts, artists have a new, re-narrative way to express art forms they increasingly pay attention to participants' experiences. Therefore, we need to consider what kinds of materials can produce the most desirable impact for those participating in my works. As a result, we must create a new relationship between participants and materials, where sensors act as a bridge between the two. In addition, we also need to show the characteristics of these materials. In my work, I hope to portray the relationship between black and white in traditional Chinese landscape painting. I aim to utilise many different materials for my project; however, following my discovery of magnetic powder, I feel that this material can be used to form a relationship with participants. 


\section{TECHNICAL APPROACHES}

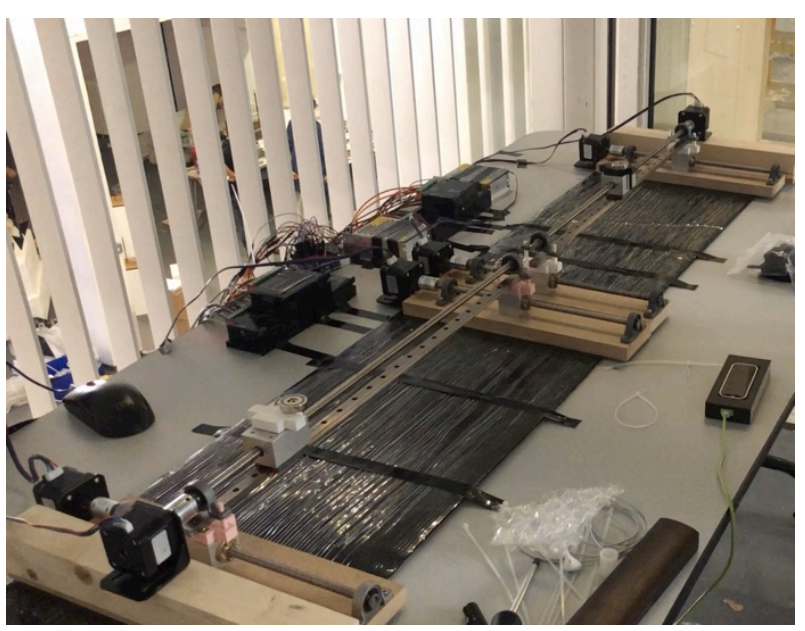

Figure 4: Installation structure.

\subsection{Physical computing}

For this project, the biggest challenge is how to incorporate physical computing into the visual arts. In the beginning of this project, some skeleton sensors were considered, such as Kinect v1 (depth camera), Real Sensor (depth camera), and Leap Motion (hand gesture sensor). In the end, the Leap Motion sensor was chosen as it allows participants to have more hand control and, at the same time, produces stable data while avoiding too many data errors.

The structures of six stepper motors are used which controls the electromagnetic position. To begin, I tried to control the movement of the position by other means, but the effect was undesirable; therefore, I switched to stepper motors, which can move efficiently and can be easily controlled by Arduino.

\subsection{Machine learning}

Machine learning algorithms have been used to make sure the installation can receive the correct action instructions and make it easier to control the participant's gesture data. This ensures the instructions for the participants can be executed with more accuracy. And therefore, it is essential to acquire new data for the use of machine learning algorithms.

In this piece of work, the data obtained from the movement of the fingers by the Leap Motion sensor, then sent to the Wekinator system - a machine learning software - for general data training. I was able to train a large amount of gesture data at once, with five classes per output. As a result, there are no irregular movements of the installation that could be caused by mechanical problems of gestures and hand movements of different age groups.
In total, the whole machine learning algorithm has ten classes, respectively corresponding to: forward, backward, left forward, right forward, left backward, right backward, eight directions, and two blank commands.

\subsection{Processing to Arduino}

The serial function of Processing (a flexible software sketchbook for visual arts) helps this project with the communication between Processing and Arduino. To begin, the Leap Motion sensor acquires original participant gesture data and sends it to the Wekinator system through OSC (Open sound Control); the Wekinator system then classifies the new data and sends it back to Processing. Once the Leap Motion sensor receives the visitor's data, it can be analysed and will allow for the motor to move.

\subsection{Physical structure}

In order to allow for the electromagnet to follow the movement of the gesture and give quick feedback, the stepper motors and lead screws will be chosen for my structure. Machine learning will also help me obtain the correct result, which will allow the structure to move to the correct position. The steppers' bracket also use 3D printed and laser cutting to build the structure.

\section{SELF-EVALUATION}

By using computational ways to represent the traditional narrative is a goal that I am trying to do. Furthermore, trying new materials and combining the two is what I need to do. Therefore, in the next step, I plan to use machine learning and material re-narrative more in computational art.

\section{REFERENCES}

Guo Xi (n.d.) Early Spring, Chinese Painting, China Online Museum.

https://www.comuseum.com/painting/masters/guoxi/early -spring/ (retrieved 13 March 2020).

Salter, C. (2015) Alien Agency. Cambridge, MA: The MIT Press.

Smelik, A. (2010). The Scientific Imaginary in Visual Culture. Goettingen: V \& R Unipress, pp.69-88.

Smigielska, M. (2019) Proteus [Vimeo] https://vimeo.com/269581453 (retrieved 12 Sep 2019).

Wiberg, M. and Robles, E. (2010) Computational Compositions: Aesthetics, Materials, and Interaction Design, International Journal of Design. http://www.ijdesign.org/index.php/IJDesign/article/vi ew/757/301 (retrieved 12 Sep 2019). 